\title{
Haemophagocytic lymphohistiocytosis syndrome in a patient with adult onset Still's disease; a diagnostic challenge
}

\author{
Madagedara R M D ${ }^{1}$, Bandara H G W A P L ${ }^{1}$, Wijethilake B H W M G T ${ }^{1}$, Athauda S D \\ Journal of the Ceylon College of Physicians, 2017, 48, 75-79
}

\section{Introduction}

Haemophagocytic lymphohistiocytosis (HLH) is a syndrome of pathologic immune activation characterized by clinical signs and symptoms of extreme inflammation ${ }^{1}$. It was described as both a familial disorder and as a sporadic one, in association with infections, malignancies, or rheumatologic disorders. HLH results from impaired functions of natural killer (NK) and cytotoxic T cells, whereas activities of lymphocytes and histiocytes are augmented, leading to phagocytosis of hematopoietic cells ${ }^{2}$. Though the primary pathogenic mechanism is linked to genetic and immunologic basis, HLH remains a multisystem disorder having a unique pattern of clinical manifestations. Secondary HLH is triggered by a variety of causative agents including infections, malignancies and rheumatological illnesses. Since the individual clinical features are non-specific, HLH and Adult Onset Stills Disease (AOSD) share overlapping clinical and a number of laboratory features making the accurate diagnosis of both syndromes difficult. HLH that arises in the course of AOSD has been reported only rarely. Tuberculosis is yet another infective cause of secondary HLH where reported cases are scanty. Here, we report two cases of secondary HLH; a young male with Still's disease and a young female with tuberculosis, both cases complicated with HLH.

\section{Case 1}

A 16-year-old patient was admitted with intermittent spiking fever up to $39.8^{\circ} \mathrm{C}$ for three weeks duration. He also had arthralgia affecting multiple large and small joints in an asymmetrical pattern without morning stiffness. He developed mild productive cough with pleuritic type of chest pain during the same period. There was a transient erythematous non-pruritic rash

\footnotetext{
${ }^{1}$ Respiratory Diseases Treatment Unit II, Teaching Hospital, Kandy, Sri Lanka.

${ }^{2}$ Haematology Unit, Teaching Hospital, Kandy, Sri Lanka.

Corresponding author: Madagedara RMD

E-mail: dmadegedara@yahoo.com
}

involving the trunk and extremities which was more prominent during fever spikes. Clinical examination revealed a morbiliform rash over the abdomen, trunk, and both arms. He was pale and had moderate hepatosplenomegaly with supra-clavicular and axillary lymphadenopathy.

Initial laboratory investigations showed haemoglobin of $5.9 \mathrm{~g} / \mathrm{dL}$, platelet count of $31 \times 10^{\%} / \mathrm{L}$, and white blood cell (WBC) count of $4.5 \times 10^{9} / \mathrm{L}$ with absolute neutrophil count of $2.7 \times 10 \%$ L. His Erythrocyte Sedimentation Rate (ESR) was $27 \mathrm{~mm}$ in $1^{\text {st }}$ hour and C- Reactive Protein (CRP) was $129 \mathrm{mg} / \mathrm{L}$. The biochemistry profile revealed aspartate aminotransferase of $42 \mathrm{U} / \mathrm{L}$, alanine aminotransferase of $62 \mathrm{U} / \mathrm{L}$, total bilirubin of $4.2 \mathrm{mg} / \mathrm{dL}$, serum ionized calcium $4.6 \mathrm{mg} / \mathrm{dL}(4-5.4 \mathrm{mg} / \mathrm{dL})$, sodium of $138 \mathrm{mEq} / \mathrm{L}$, potassium of $4.5 \mathrm{mEq} / \mathrm{L}$, creatinine of $0.8 \mathrm{mg} / \mathrm{dL}$, glucose of $109 \mathrm{mg} / \mathrm{dL}$, albumin of $3.9 \mathrm{~g} / \mathrm{dL}$, and total protein of $5.7 \mathrm{~g} / \mathrm{dL}$. A chest radiograph revealed hilar lymphadenopathy, bibasal ground glass appearance with patchy consolidation (Figure 1).

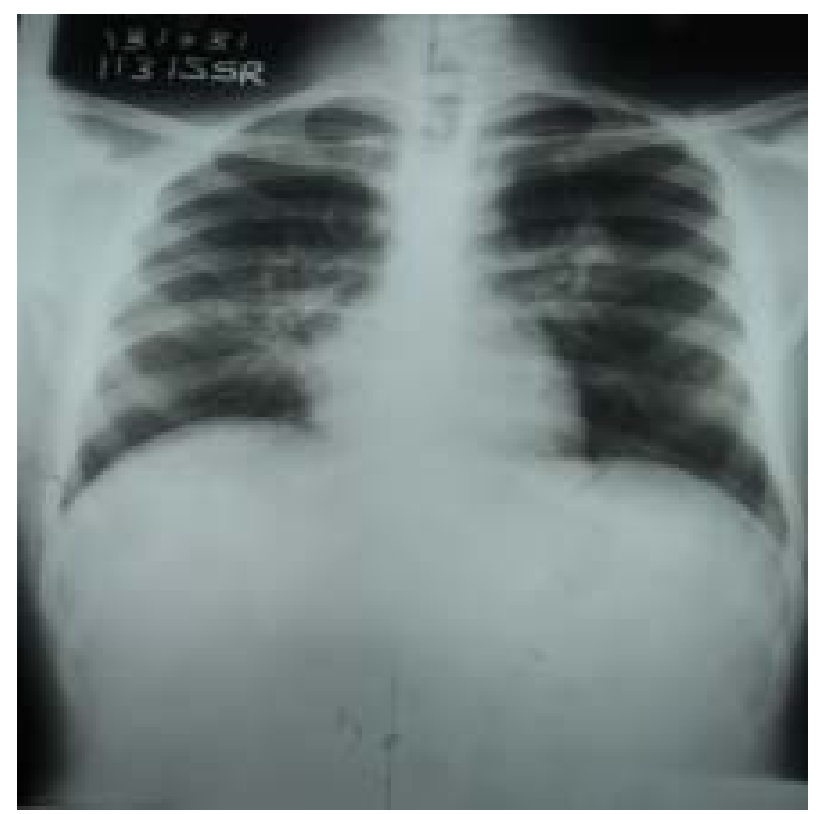

Figure 1. Chest X-ray of the patient demonstrated pulmonary infiltrates. 
A computed tomography (CT) scan of the chest revealed bilateral perihilar patchy consolidation with mediastinal lymphadenopathy and bilateral small pleural effusion (Figure 2). Abdominal cuts of the chest CT showed multiple hypodense infiltrative lesions over the spleen (Figure 3). Lymph node biopsy showed only nonspecific reactive changes with no definitive evidence of tuberculosis (TB), sarcoidosis or infiltration by lymphoma or leukaemic cells. Microbiological investigations included repeatedly negative blood cultures, negative sputum cultures and negative smear for Acid Fast Bacilli and Pneumocystis. Mantoux test, gamma interferon assay, serology for Human Immuno deficiency Virus (HIV), Ebstein-Barr virus (EBV), Cytomegalovirus (CMV), Mycoplasma and Brucella also were negative. He had a normal serum Angiotensin Converting Enzyme (ACE) level. Antinuclear Antibody (ANA), Antibodies for Double strand DNA (dsDNA), Rheumatoid factor (RF), C and P Anti-Neutrophil Cytoplasmic Antibodies (ANCA) were negative.

Intravenous broad-spectrum antibiotics were commenced and anti-tuberculosis therapy was added to the antibiotic regimen as there was a high clinical suspicion of TB. He continued to have fever and worsening of pancytopenia.

The combination of fever, arthralgia, transient nonpruritic rash, hepato-splenomegaly and lymphadenopathy with negative ANA and rheumatoid factor fulfilled the Yamaguchi criteria for AOSD. Subsequent investigations showed a serum ferritin of $>30,000 \mathrm{ng} / \mathrm{mL}$ which further supported the diagnosis.

However, the worsening pancytopenia was not compatible with the diagnosis of AOSD. Subsequently he was subjected to a bone marrow trephine biopsy, which revealed increased haemophagocytosis (Figure 4). This finding was suggestive of the diagnosis of $\mathrm{HLH}$.

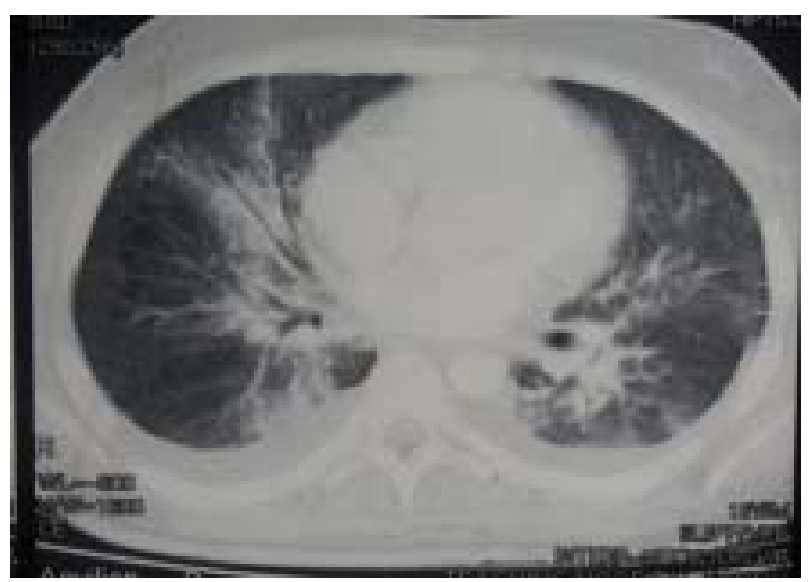

Figure 2. CT scan of the chest showed pulmonary infiltrates, hilar lymphadenopathy and pleural effusion.
Our patient fulfilled the diagnostic criteria of fever, splenomegaly, cytopenia, haemophagocytosis in bone marrow and high serum ferritin. He was found to have high Triglyceride (TAG) level of $558.3 \mathrm{mg} / \mathrm{dL}$ (10-200 $\mathrm{mg} / \mathrm{dL}$ ) and high Lactate dehydrogenase (LDH) which further reinforce the diagnosis of $\mathrm{HLH}$.

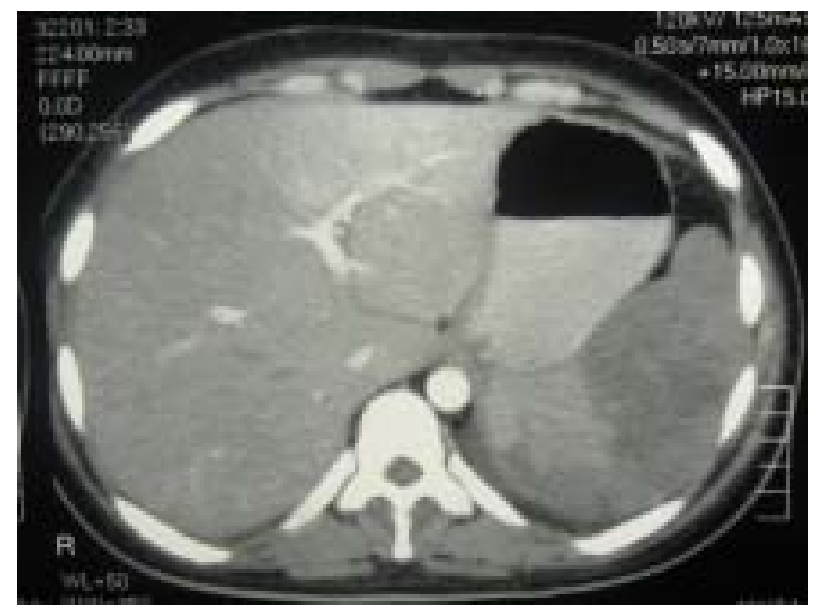

Figure 3. CT scan of the abdomen demonstrated multiple splinic infiltrates.

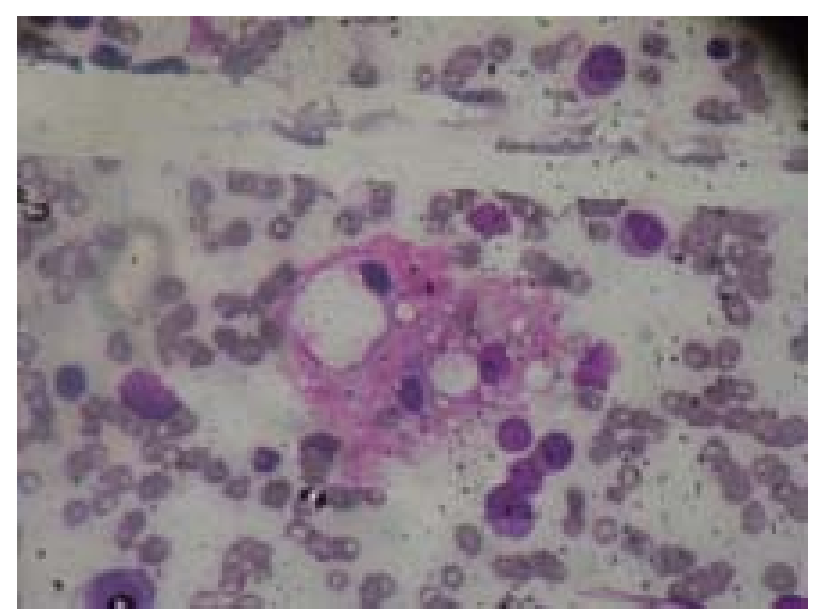

Figure 4. Bone marrow biopsy demonstrated increased haemophagocytosis.

The ongoing treatment was modified by adding high dose of oral steroids (dexamethasone), oral etoposide in addition to antibiotics. Patient had a remarkable recovery in both clinical and haematological parameters (Graph 1 to 3).

\section{Case 2}

A 17-year-old female was admitted with fever for more than 7 days. She also had abdominal pain, irritability, malaise and anorexia. She did not have any skin rashes, joint involvement, bleeding diatheses. She was pale, did not have lymphadenopathy or hepatosplenomegaly. 


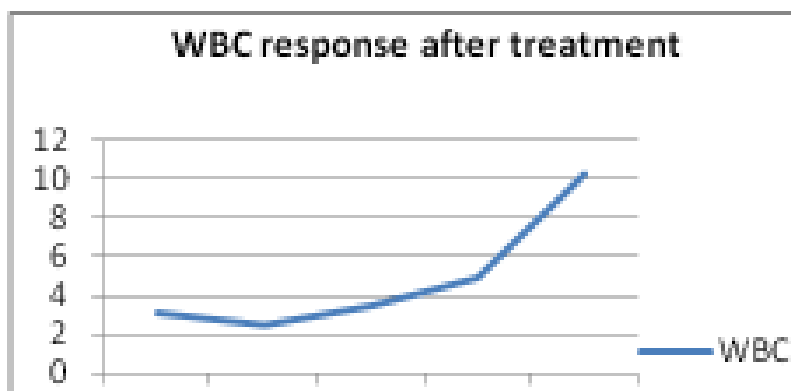

Day 1 Day 3 Day 5 Day 7 Day 10

Graph 1. WBC response after treatment commenced on Day 7.

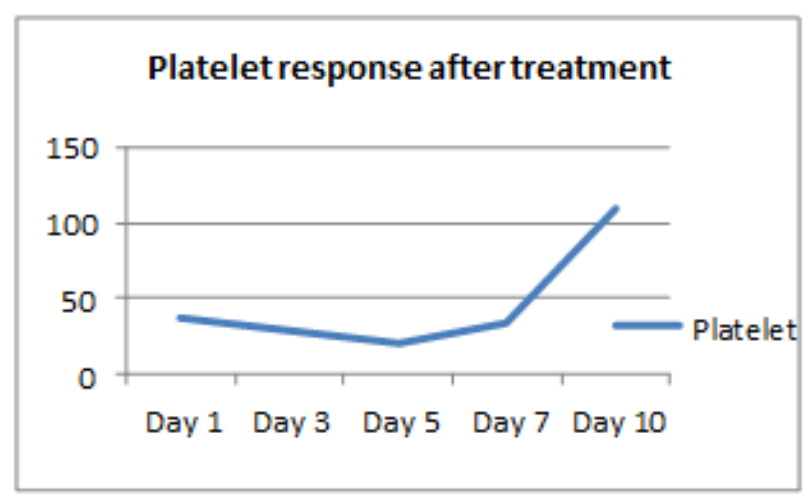

Graph 2. Platelet response after treatment commenced on Day 7.

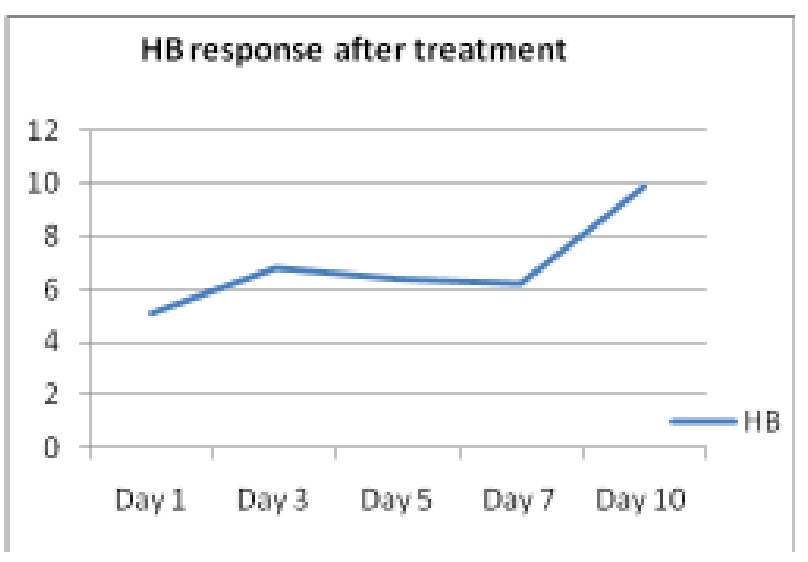

Graph 3. HB response after treatment commenced on Day 7.

Initial laboratory investigations revealed Haemoglobin of $6.7 \mathrm{~g} / \mathrm{dl}$, White blood cell (WBC) count of $4.5 \times 10^{9} / \mathrm{L}$ with $72 \%$ neutrophils; absolute neutrophil count $3.2 \times 10^{9} / \mathrm{L}$ and Platelet count of $63 \times 10^{9} / \mathrm{L}$. Erythrocyte Sedimentation Rate (ESR) was 131 in the $1^{\text {st }}$ hour and C-Reactive Protein (CRP) was $103 \mathrm{mg} / \mathrm{L}$. Biochemical profile revealed serum sodium of $138 \mathrm{mmol} /$
L, potassium $3.3 \mathrm{mmol} / \mathrm{L}$, blood urea $2.02 \mathrm{mmol} / \mathrm{L}$, serum creatinine $0.5 \mathrm{mg} / \mathrm{dl}$, aspartate aminotransferase (AST) $54.3 \mathrm{U} / \mathrm{L}$, alanine aminotransferase (ALT) $36.8 \mathrm{U} / \mathrm{L}$, fasting blood sugar (FBS) $3.2 \mathrm{mmol} / \mathrm{L}$, serum albumin $3.8 \mathrm{~g} / \mathrm{dL}$ with total protein of $6.66 \mathrm{~g} / \mathrm{dL}$. Alkaline phosphatase level was $229 \mathrm{U} / \mathrm{L}$. Serum triglyceride level was $588.3 \mathrm{mg} / \mathrm{dL}$ and serum ferritin level was 4479.12 $\mathrm{ng} / \mathrm{mL}$.

Chest radiograph and USS Abdomen were normal.

Microbiological investigations showed both blood and urine cultures to be negative. Mycoplasma serology was 1:80 positive. Retro-viral screen was negative. Mantoux test reading was $11 \mathrm{~mm}$ and sputum smear for Acid Fast Bacilli (AFB) was negative. Sputum culture AFB was negative.

Urine full report revealed trace protein with 1-2 pus cells per high power field. Urine dipstick for hCG was negative. Rheumatoid factor and Antinuclear Antigen (ANA) were negative.

Initial Blood picture was consistent with features of iron deficiency anaemia and first bone Marrow Biopsy was consistent with bacterial infection/ inflammation. The Second Bone Marrow Biopsy showed typical Haemophagocytosis (Figure 5).

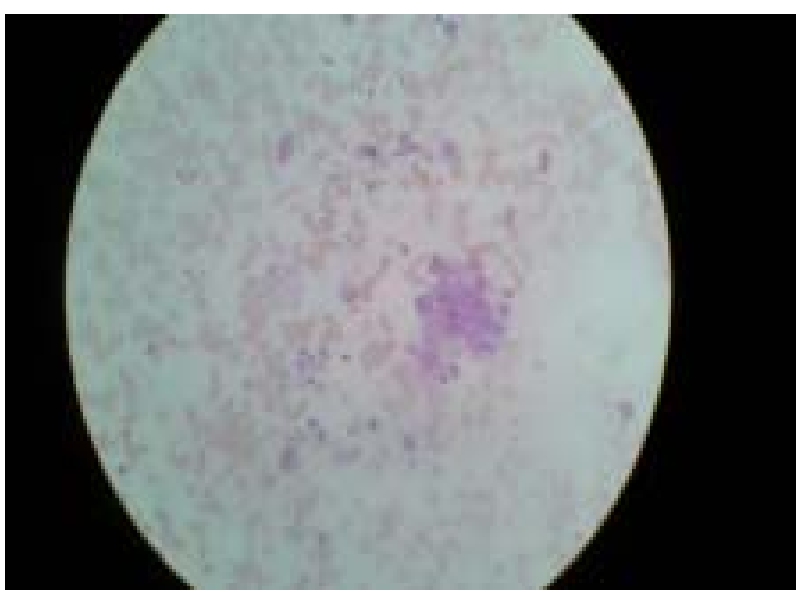

Figure 5. Bone marrow biopsy demonstrated typical haemophagocytosis.

Initial treatment with broad spectrum antibiotics was unsuccessful.

This patient fulfilled five criteria out of eight introduced by Henter et al. in diagnosing Haemophagocytic Lymphohistocytosis. Patient had fever, cytopenia, haemophagocytosis in bone marrow, hypertriglyceridaemia and hyperferritinaemia.

Sri Lanka being an intermediate risk burden country for tuberculosis and the presentation with 
persistent fever and positive Mantoux test, the patient was started on anti-tuberculosis chemotherapy as for smear negative pulmonary tuberculosis. She was also started on high dose intravenous steroids tailing off over 8 weeks, etoposide for 8 weeks, Pneumocyctis cariini prophylaxis with co-trimoxazole $960 \mathrm{mg}$ bd and anti-fungal prophylaxis with fluconazole $100 \mathrm{mg}$ bd. Patient showed a remarkable clinical, biochemical and haematological improvement (Graph 4,5,6).

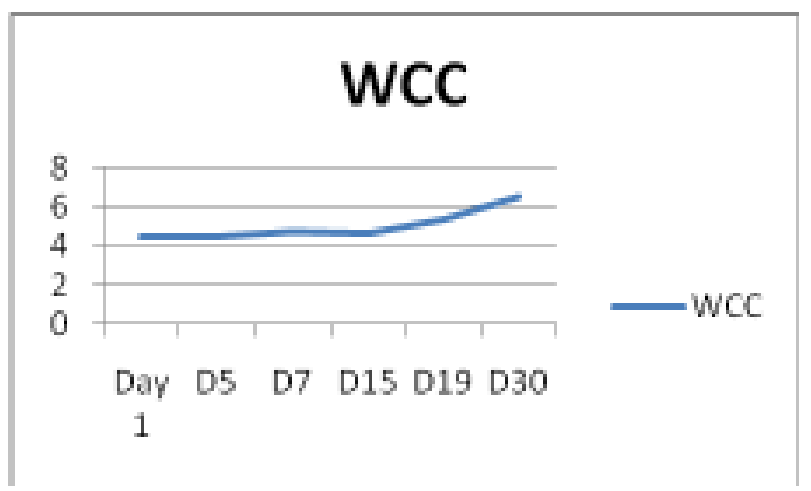

Graph 4. White Cell Count response after treatment commenced

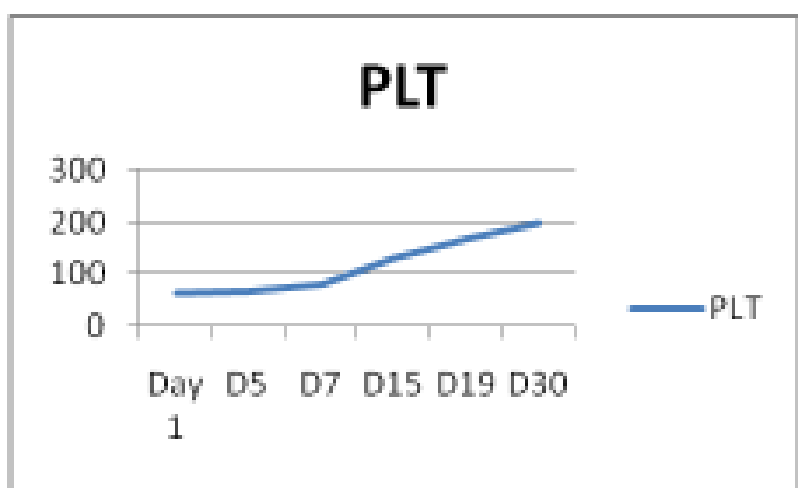

Graph 5. Platelet response after treatment commenced.

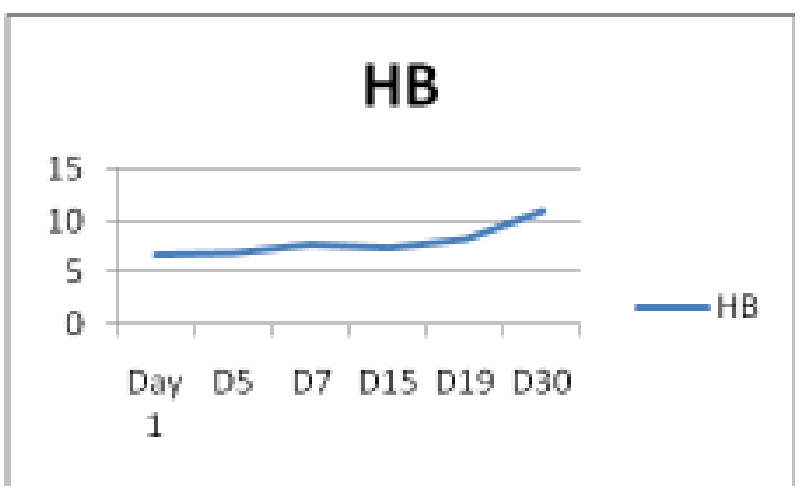

Graph 6. Haemoglobin response after treatment.

\section{Discussion}

$\mathrm{HLH}$ syndrome is an activation of mononuclear phagocyte system cells, with hemophagocytosis in bone marrow and the rest of reticulo-endothelial system. This syndrome can be either primary or reactive (secondary) ${ }^{3}$. Genetic HLH has an autosomal recessive inheritance pattern, and usually arises in infants ( $80 \%$ cases); however, in rare cases it can also occur in adults ${ }^{4}$ and is also associated with impaired NK cell function ${ }^{5}$.

Secondary HLH has a better outcome than primary $\mathrm{HLH}$. It can be triggered mainly by viral infections (especially EBV) ${ }^{6}$ and also by bacterial, parasitic or fungal infections. It can also develop in malignancies and rheumatoid disorders ${ }^{7}$.

Secondary HLH and AOSD share several clinical and laboratory features, including high fever, hepatosplenomegaly, lymphadenopathy, liver injury, hyperferritinaemia and coagulopathy, which may explain the difficulty in recognizing $\mathrm{HLH}$ complicating the flare of AOSD. The main difference between the two diseases is cutaneous and articular involvement, which is a common presentation in AOSD and is uncommon in secondary $\mathrm{HLH}$. In our patient the evidence of relatively low ESR compared to high CRP, leucopenia and hypertriglyceridemia also supports the diagnosis of $\mathrm{HLH}$ over straightforward AOSD. Raised serum triglyceride level is considered to be a good marker of haemophagocytic syndrome ${ }^{8}$. In our patient, serum triglycerides were markedly raised during the acute phase of the illness and it could be used as a differentiating feature for HLH diagnosis.

The diagnostic challenge arises when the features of both of the disorders overlap with one or more features of sepsis, which could be a deadly coexistence. Here, we were able to exclude possible sepsis by demonstrating repeatedly negative blood cultures for bacteria and fungi, negative serology for HIV, Hepatitis B/C, EBV, CMV and Brucella and negative smears and cultures for TB. Next diagnostic challenge arises, in cases of secondary $\mathrm{HLH}$, finding the causative trigger. In this scenario, we were able to exclude as much as possible the possibility of sepsis, tuberculosis, autoimmune disease, vasculitis and hematological malignancies.

Since our patient fulfilled criteria for AOST and the bone marrow finding strengthen the necessary criteria for $\mathrm{HLH}$, the reactive HLH secondary to AOSD was made. Extensive studies could not identify any evidence of viral infection or other known underlying disorders associated with reactive HLH.

Although the features of secondary $\mathrm{HLH}$ and AOSD are well characterized, the underlying 
physiopathology is not well understood. The most consistent immunological abnormality described in patients with primary or secondary haemophagocytic syndrome is impairment of cytotoxic function ${ }^{9}$. The deficient cytotoxic function may bring about failure to provide complete pathogen destruction and persistent lymphocyte and macrophage activation ${ }^{10}$. Sustained macrophage activation may result in tissue infiltration, production of ferritin and high levels of tumour necrosis factor a (TNFa) and interleukin (IL), IL-6, IL-18, IL-8, observed in flares of AOSD and $\mathrm{HLH}^{11}$. Highly activated macrophages are thought to have a key role in the pathogenesis of $\mathrm{AOSD}^{12}$. Hence, sustained macrophage activation in AOSD may lead to reactive $\mathrm{HLH}$ after a sudden intensification of disease activity.

Pulmonary involvement is well-known though rare in AOSD and is seen in up to $53 \%$ of cases, with the most common pulmonary diseases being pleural effusion and transient pulmonary infiltrates ${ }^{13}$ as seen in our patient. As our patient had pulmonary infiltrates in a background of pancytopenia, exclusion of opportunistic infection was the next diagnostic challenge.

Tuberculosis remains a health burden in the South East Asia with Sri Lanka recognized as an intermediate risk country. Mycobacterium tuberculosis (MTB) has a diverse variety of clinical manifestations. $\mathrm{HLH}$ is an uncommon yet a potentially fatal complication of tuberculosis with unpredictable clinical course. In patients with tuberculosis, cytopenia, organomegaly and coagulopathy should alert the clinician to consider secondary $\mathrm{HLH}^{14}$.

MTB, being an obligate intracellular pathogen, is able to aggravate $\mathrm{Th}_{1}$ cell-mediated cytotoxicity and macrophage overactivity that can lead to HLH in susceptible patients. This is supported by increased serum levels of IFN- $\gamma, \mathrm{M}-\mathrm{CSF}$, and TNF- $\alpha$ in patient with tuberculosis ${ }^{14}$.

Since there is no consensual treatment for $\mathrm{HLH}$ in AOSD, we planned out our management according to the HLH 2004 treatment protocol. He was started on oral dexamethesone to suppress the sever inflammation, etoposide to restrain the over-stimulated antigen-presenting cells, which are the macrophages with close monitoring of the clinical and haematological parameters. He achieved a good clinical and haematological recovery.

In the treatment of tuberculosis complicated with $\mathrm{HLH}$ immunomodulatory therapy is a debated field but it is advocated to use anti-tuberculous therapy (ATT) early. HLH complicating tuberculosis is known to have high morbidity and mortality with or without ATT ${ }^{14}$.

\section{References}

1. Michael B. Jordan, Carl E. Allen, Sheila Weitzman, Alexandra H. Filipovich, Kenneth L,McClain. How we treat hemophagocytic lymphohistiocytosis. Blood 2013: 1-26.

2. Rouphael NG, Talati NJ, Vaughan C, Cunningham K, Moreira $\mathrm{R}$, Gould C. Infections associated with haemophagocytic syndrome. Lancet Infectious Disease 2007; 7: 814-22.

3. Juan Mayordomo-Colunga, Corsino Rey, Soledad González and Andrés Concha. Multiorgan failure due to hemophagocytic syndrome: A case report. Cases Journal 2008, 1: 209.

4. Maciej Machaczka, Wojciech Sydor, Machgorzata Rucinska, Marta Szostek and Jacek Musial. AutoimmuneAssociated Hemophagocytic Syndrome /Macrophage Activation Syndrome. Autoimmune Disorders - Current Concepts and Advances from Bedside to Mechanistic Insights 2011; 80-98.

Available from: http://www.intechopen.com

5. Gritta Janka and Udo zur Stadt. Familial and Acquired Hemophagocytic Lymphohistiocytosis. American Society of Hematology 2005: 82-8.

6. Janka GE. Hemophagocytic syndromes. Blood Rev 2007; 21: 245-53.

7. Stabile A, Bertoni B, Ansuini V, La Torraca I, Salli A, Rigante D. The clinical spectrum and treatment options of macrophage activation syndrome in the pediatric age. European Review for Medical and Pharmacological Sciences 2006; 10: 53-59.

8. Karras A, Hermine O. Syndrome d'activation macrophagique [Macrophage activation syndrome]. Rev Med Interne 2002; 23: $768-78$.

9. Grom AA. Macrophage activation syndrome and reactive hemophagocytic lymphohistiocytosis. Current Opinionin Rheumatology 2003; 1: 587-90.

10. Wulffraat NM, Rijkers GT, Elst E, Brooimans R, Kuis W. Reduced perforin expression in systemic juvenile idiopathic arthritis is restored by autologous stem-cell transplantation. Rheumatology 2003; 42: 375-9.

11. Villanueva J, Lee S, Giannini EH, Graham TB, Passo MH, Filipovich A, et al. Natural killer cell dysfunction is a distinguishing feature of systemic onset juvenile rheumatoid arthritis and macrophage activation syndrome. Arthritis Research and Therapy 2005; 7: R30-7.

12. Amenomori M, Migita K, Miyashita T, Yoshida S, Ito M, Eguchi $\mathrm{K}$, et al. Cytomegalovirus-associated hemophagocytic syndrome in a patient with adult onset Still's disease. Clinical and Experimental Rheumatology 2005; 23: 100-2.

13. Cheema GS, Quismorio FP Jr. Pulmonary involvement in adult-onset Still's disease. Current Opinionin Pulmonary Medicine 1999; 5: 305-9.

14. Padhi S, Ravichandran K, Sahoo J, Vargese R, Basheer A. Haemophagocytic lymphohistiocytosis: an unusual complication in disseminated Mycobacterium Tuberculosis. Lung India 2015; 32(6): 593-601. 